\title{
Familiares vivenciando cuidados paliativos de crianças com câncer hospitalizadas: uma revisão integrativa
}

\author{
Families experiencing palliative care for hospitalized children with cancer: an integrative review
}

Familiares vivenciando cuidados paliativos de niños hospitalizados con cáncer: una revisión integradora

\author{
Cristineide dos Anjos' ${ }^{\circledR}$; Rose Mary Costa Rosa Andrade Silva' $\odot$; Eliane Ramos Pereiral $\odot$;

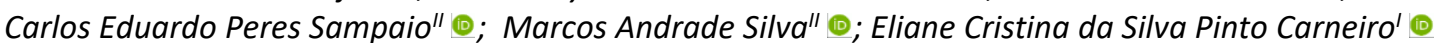

'Universidade Federal Fluminense, Niterói, RJ, Brasil; "Universidade do Estado do Rio de Janeiro, Rio de Janeiro, RJ, Brasil

\begin{abstract}
RESUMO
Objetivo: analisar a produção do conhecimento sobre a experiência da família acerca dos cuidados paliativos da criança com câncer hospitalizada na unidade de terapia intensiva e discutir o papel da enfermagem no atendimento à família da criança com câncer frente aos cuidados paliativos. Método: revisão integrativa realizada na PUBMED, LILACS, SCOPUS, SCIELO e CINAHL entre janeiro e março de 2020. Resultados: a amostra foi composta por 13 artigos, originando duas categorias: $O$ impacto do cuidado paliativo da criança com câncer na dinâmica familiar e o cuidado multiprofissional, em especial dos enfermeiros, à família da criança com câncer. Conclusão: os estudos revelaram o panorama nacional e internacional dos cuidados paliativos à criança com câncer e a desestruturação da dinâmica familiar nos aspectos físicos, sociais, psicológicos, e financeiros, caracterizando o período como estressante e doloroso. Os enfermeiros estabelecem condutas terapêuticas objetivando promover qualidade de vida para crianças em cuidados paliativos e seus familiares.
\end{abstract}

Descritores: Criança; Família; Câncer; Cuidados Paliativos; Unidades de Terapia Intensiva.

\begin{abstract}
Objective: to examine production of knowledge on families' experience of palliative care for children with cancer hospitalized in an intensive care center and discuss the role of nursing in assisting the family of the child in palliative cancer care. Method: this integrative review was conducted on PubMed, LILACS, Scopus, SciELO and CINAHL between January and March 2020. Results: from the sample of 13 articles, two categories originated: The impact of palliative cancer care for children on family dynamics and multiprofessional care, especially by nurses, for the family of the child with cancer. Conclusion: the studies revealed the national and international panorama of palliative care for children with cancer and the breakdown of physical, social, psychological, and financial family dynamics, characterizing the period as stressful and painful. Nurses establish therapeutic approaches aimed at promoting quality of life for children in palliative care and their families.

Descriptors: Child; Family; Cancer; Palliative Care; Intensive Care Units.
\end{abstract}

\section{RESUMEN}

Objetivo: analizar la producción de conocimiento sobre la experiencia familiar en los cuidados paliativos de niños con cáncer, hospitalizados en la unidad de cuidados intensivos, y discutir el papel de la enfermería en la asistencia a la familia del niño con cáncer frente a los cuidados paliativos. Método: Revisión integradora realizada en PUBMED, LILACS, SCOPUS, SCIELO y CINAHL entre enero y marzo de 2020. Resultados: La muestra estuvo conformada por 13 artículos, originando dos categorías: El impacto de los cuidados paliativos para niños con cáncer en la dinámica familiar y los cuidados multiprofesionales, especialmente de las enfermeras, a la familia del niño con cáncer. Conclusión: Los estudios revelaron el panorama nacional e internacional de los cuidados paliativos para niños con cáncer y la ruptura de la dinámica familiar en aspectos físicos, sociales, psicológicos y económicos, caracterizándolo como un período estresante y doloroso. Las enfermeras establecen enfoques terapéuticos dirigidos a promover la calidad de vida de los niños en cuidados paliativos y sus familias.

Descriptores: Niño; Familia; Cáncer; Cuidados Paliativos; Unidades de Cuidados Intensivos.

\section{INTRODUÇÃO}

O câncer infantil corresponde a um grupo de várias doenças que têm em comum a proliferação descontrolada de células anormais e que pode ocorrer em qualquer local do organismo. Os tumores mais frequentes na infância e na adolescência são as leucemias, os do sistema nervoso central e (os) linfomas. Além de tumores como o neuroblastoma, Wilms, retinoblastoma, germinativo, osteossarcoma e sarcomas ${ }^{1}$.

Estima-se que o número de casos novos de câncer infanto-juvenis esperados para no Brasil, para cada ano do triênio 2020-2022, será de 4.310 novos casos no sexo masculino e de 4.150 para o sexo feminino. Esses valores correspondem a um risco estimado de 137,87 novos casos por milhão no sexo masculino e de 139,04 por milhão para o sexo feminino ${ }^{2}$.

Autora correspondente: Cristineide dos Anjos. E-mail: cristineideminuzzi@yahoo.com.br Editora Científica: Cristiane Helena Gallasch; Editor Associado: Antonio Marcos Tosoli Gomes 
Assim, o câncer é a doença que mais mata crianças e adolescente, sendo a segunda maior causa de óbito neste grupo etário, superada somente pelos acidentes e mortes violentas. No Brasil, em 2017, ocorreram, para o sexo masculino, 1.467 óbitos por câncer de infanto-juvenis para todas as neoplasias, com um risco estimado de 44,46/milhão. Já para o sexo feminino, houve 1.086 óbitos $(34,30 / \text { milhão })^{1,2}$.

O diagnóstico e a experiência com o câncer apresentam-se como um longo caminho a ser percorrido pela criança ou adolescente e por sua família, com repercussões diversas no seu cotidiano, considerando as conseqüências precoces e tardias do tratamento. As diferentes modalidades terapêuticas no campo da oncologia oferecem novas chances de cura à criança e ao adolescente, abrindo-lhes novas perspectivas de vida ${ }^{3}$.

Dessa forma, quando a doença é diagnosticada precocemente, existem grandes chances de tratamento e cura. No entanto, a demora no diagnóstico pode comprometer o tratamento, evoluindo, dessa forma, para o cuidado paliativo exclusivo.

A Organização Mundial de Saúde (OMS) define cuidados paliativos como uma abordagem que visa melhorar a qualidade de vida dos pacientes e de suas famílias, realizados por meio da prevenção e alívio do sofrimento, pela identificação precoce, avaliação correta e tratamento da dor e de outros problemas de ordem física, psicossocial e espiritual. Reformulado, anos depois, na parte que diz que deve ser iniciada desde o momento do diagnóstico da doença e que deve perdurar por toda a vida da criança até sua morte e depois dela ${ }^{4}$.

Os cuidados paliativos constituem abordagem de cuidado diferenciada com enfoque no cuidado da criança com câncer em fase terminal, abordando a importância dos cuidados paliativos, incorporados no cuidado de enfermagem diário a essa criança desde a definição do diagnóstico até o final da vida, a partir da atenção holística aos aspectos físico, psicológico, social e espiritual da criança ${ }^{5}$.

Dessa forma, o propósito assistencial nesse âmbito não é buscar a cura da neoplasia, mas oferecer um cuidado interdisciplinar objetivando fornecer suporte, informação e conforto para pacientes com doença ameaçadoras da vida e seus familiares. Atualmente, calcula-se que 20 milhões de pessoas/ano, sendo $6 \%$ dessas crianças, teriam indicação para cuidados paliativos ${ }^{6}$.

No entanto, durante as hospitalizações existe a possibilidade de a criança necessitar de um centro de terapia intensivo e, nessa unidade, os profissionais da saúde ocupam lugares e papéis distintos, participando do atendimento ao paciente com risco de vida e/ou eminência de morte. Nesses casos, admitem-se diferentes desfechos, em que o paciente pode sair recuperado ou, em outros casos, apenas prorrogar o seu tempo de vida ${ }^{7,8}$.

A disfunção familiar, em decorrência da hospitalização em terapia intensiva, é vivenciada por todos os seus membros e pode ser amplificada pelas incertezas que acompanham as mudanças críticas e emergentes na saúde da criança, sendo possível identificar uma série de fontes de estresse, incluindo a insegurança relacionada ao ambiente da terapia intensiva, que envolve alarmes e equipamentos e a comunicação com a equipe, aos papéis do cuidador, a condição da criança e as alterações ocasionadas pela doença ${ }^{9}$, principalmente quando a criança se encontra em cuidados paliativos exclusivos nessa unidade.

Na literatura científica há diversos estudos que destacam os benefícios da participação da família no cuidado à criança hospitalizada. Porém, no contexto da experiência de familiares de crianças com câncer em cuidados paliativos exclusivos na unidade de terapia intensiva oncológica ainda não se adquiriu visibilidade na literatura. A complexidade desse tema, aliado à escassez de literatura e pesquisas, justifica a realização deste estudo.

Portanto, este tema possui sua importância na medida em que os cuidados paliativos surgem como a condição básica para resgatar o respeito e a dignidade daquele que tem doença, principalmente considerando-se o ambiente de UTI, por ser um setor que recebe pacientes graves e muitas vezes prestes a falecerem.

Assim foram adotados como objetivos: analisar a produção do conhecimento sobre a experiência da família acerca dos cuidados paliativos da criança com câncer hospitalizada na unidade de terapia intensiva e discutir o papel da enfermagem no atendimento à família da criança com câncer frente aos cuidados paliativos.

\section{MÉTOdO}

Trata-se de uma revisão integrativa de literatura que tem como propósito reunir achados de estudos desenvolvidos com diferentes metodologias, permitindo aos revisores fazer a análise e a síntese dos dados primários de forma sistemática e rigorosa ${ }^{10}$.

Para a construção dessa revisão, foram percorridas as seis etapas que consistem em: 1) elaboração da questão de pesquisa; 2) busca na literatura dos estudos primários com base nos critérios de inclusão e exclusão; 3) organização dos estudos pré-selecionados (extração de dados dos estudos); 4) análise crítica dos estudos selecionados; 5) síntese dos resultados; e 6) apresentação da revisão integrativa ${ }^{11}$. 
Para a elaboração da pergunta de pesquisa, utilizou-se a estratégia mnemônica PICo ${ }^{12}$ (População: criança/câncer/família; Intervenção: Cuidados Paliativos; Contexto: hospitalização no centro de terapia intensiva). Após o uso da estratégia descrita, foi possível elaborar a seguinte questão norteadora: Qual a produção do conhecimento sobre a experiência da família acerca dos cuidados paliativos da criança com câncer hospitalizada na unidade de terapia intensiva, e o papel da enfermagem no atendimento à família da criança com câncer frente aos cuidados paliativos?

A seleção dos artigos ocorreu de janeiro a março de 2020 utilizando as bases de dados eletrônicas, PUBMED (National Library of Medicine), LILACS (Literatura Latino-Americana e do Caribe em Ciências da Saúde), SCIELO (Scientific Electronic Library Online), SCOPUS (SciVerse Scopus) e CINAHL (Cummulative Index to Nursing and Allied Health Literature). A terminologia utilizada para busca baseou-se no Medical Subject Headings (MESH) e nos Descritores em Ciências da Saúde (DECS). Cada descritor controlado do elemento PICo ${ }^{12}$ foi cruzado usando o operador boleano AND resultando no seguinte cruzamento com base na estratégia PICo ${ }^{12}$. P = Criança/ child AND câncer/neoplasm AND família/Family; I = Cuidados paliativos/palliative care; Co = Centro de terapia intensiva/ intensive care unit.

Os critérios de inclusão foram: publicações de 2015 a 2020, artigos completos disponíveis para acesso, produções em inglês, espanhol e português, que envolvessem familiares de crianças em idade pré-escolar, escolar e adolescente com câncer em cuidados paliativos hospitalizados, realizados por pesquisadores da área da saúde e que apresentasse contextualização referente ao assunto investigado. Foram excluídas as publicações que abordavam neonatos e lactentes, duplicadas e que não estavam alinhadas aos critérios de inclusão, além de artigos de revisão integrativa, de opinião, editoriais, teses e dissertações.

A seleção dos estudos foi realizada, inicialmente, pela leitura dos títulos e resumos, com base nos critérios de inclusão, totalizando 222 artigos, sendo que 28 publicações foram consideradas potencialmente elegíveis e selecionadas para leitura na íntegra, das quais 13 foram incluídas na amostra final desta revisão integrativa.

Para sistematizar o processo de inclusão dos estudos optou-se pela metodologia PRISMA ${ }^{13}$. A seleção dos artigos é apresentada em um diagrama de fluxo (Figura 1).

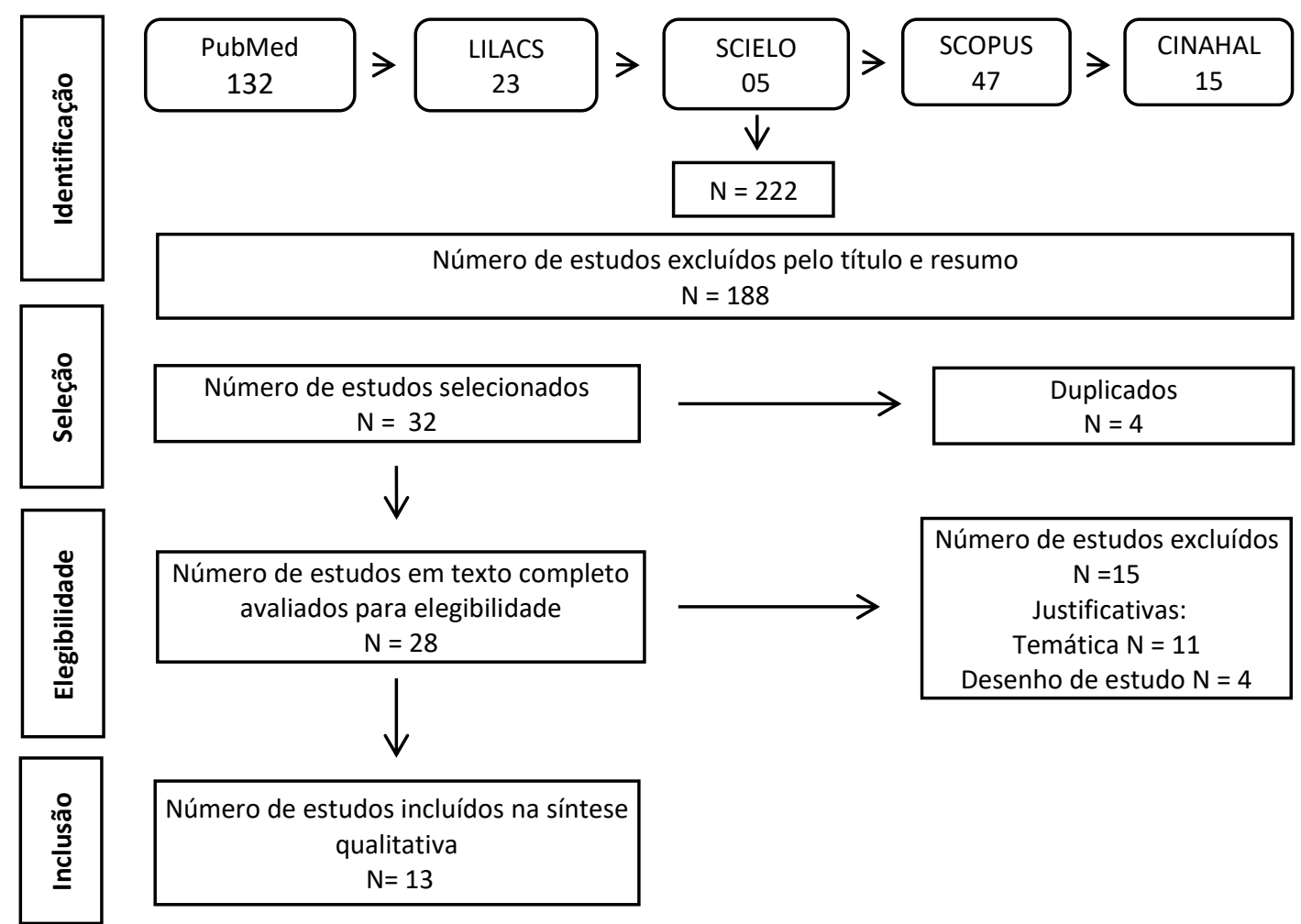

FIGURA 1: Fluxo de seleção dos artigos de revisão, segundo o PRISMA. Rio de Janeiro, Brasil, 2020. 


\section{RESULTADOS}

A síntese dos artigos foi agrupada nas Figuras 2 e 3, referentes à caracterização por título, ano, local, base, objetivos, método do estudo/participantes e contribuições.

\begin{tabular}{|c|c|c|c|c|}
\hline Título & $\begin{array}{l}\text { Ano, Local, } \\
\text { Base }\end{array}$ & Objetivos & $\begin{array}{l}\text { Metodologia/ } \\
\text { participantes }\end{array}$ & Contribuições \\
\hline $\begin{array}{l}\text { Vivências de mães de } \\
\text { crianças com câncer em } \\
\text { cuidados paliativos mediante } \\
\text { diagnóstico, tratamento e } \\
\text { apoio familiar }{ }^{15} \text {. }\end{array}$ & $\begin{array}{l}2020 \\
\text { BRASIL } \\
\text { LILACS }\end{array}$ & $\begin{array}{l}\text { Investigar a vivência de mães } \\
\text { de crianças com câncer em } \\
\text { cuidados paliativos acerca do } \\
\text { diagnóstico e tratamento. }\end{array}$ & $\begin{array}{l}\text { Estudo qualitativo } \\
\mathrm{N}=10 \text { mães }\end{array}$ & $\begin{array}{l}\text { Enfatizar que os cuidados } \\
\text { paliativos precisam ser inseridos } \\
\text { integralmente na assistência em } \\
\text { oncologia pediátrica. }\end{array}$ \\
\hline $\begin{array}{l}\text { Parent perspectives of } \\
\text { receiving early information } \\
\text { about palliative and end-of- } \\
\text { life care options from their } \\
\text { child's pediatric providers }{ }^{16}\end{array}$ & $\begin{array}{l}2019 \\
\text { Estados } \\
\text { Unidos } \\
\text { PUBMED }\end{array}$ & $\begin{array}{l}\text { Descrever as perspectivas } \\
\text { dos pais sobre o recebimento } \\
\text { de cuidados paliativos } \\
\text { precoces. }\end{array}$ & $\begin{array}{l}\text { Estudo qualitativo } \\
\mathrm{N}=10 \text { pais }\end{array}$ & $\begin{array}{l}\text { Encorajar os pais a respeito de } \\
\text { metas realistas esperadas para } \\
\text { a condição e cuidados de seus } \\
\text { filhos em cuidados paliativos. }\end{array}$ \\
\hline $\begin{array}{l}\text { Predictors of location of } \\
\text { death for children with } \\
\text { cancer enrolled on a } \\
\text { palliative care service }{ }^{17}\end{array}$ & $\begin{array}{l}2018 \\
\text { Estados } \\
\text { Unidos } \\
\text { CINAHAL }\end{array}$ & $\begin{array}{l}\text { Identificar subgrupos } \\
\text { preventivamente de } \\
\text { pacientes com maior risco de } \\
\text { morrer na UTIP. }\end{array}$ & $\begin{array}{l}\text { Estudo de coorte } \\
\text { retrospectivo } \\
\mathrm{N}=321\end{array}$ & $\begin{array}{l}\text { Promover intervenções } \\
\text { direcionadas a subgrupos de } \\
\text { risco, aperfeiçoando os } \\
\text { cuidados a crianças com câncer } \\
\text { e suas famílias na UTIP. }\end{array}$ \\
\hline $\begin{array}{l}\text { Experiências de mães de } \\
\text { crianças com câncer em } \\
\text { cuidados paliativos }^{18} \text {. }\end{array}$ & $\begin{array}{l}2018 \\
\text { Brasil } \\
\text { LILACS }\end{array}$ & $\begin{array}{l}\text { Compreender experiências } \\
\text { de mães de crianças com } \\
\text { câncer em cuidados } \\
\text { paliativos. }\end{array}$ & $\begin{array}{l}\text { Estudo Qualitativo } \\
\mathrm{N}=20\end{array}$ & $\begin{array}{l}\text { Contribuir para a construção de } \\
\text { um novo olhar acerca da } \\
\text { vivência das mães de crianças } \\
\text { com câncer. }\end{array}$ \\
\hline $\begin{array}{l}\text { Patients' and parents' views } \\
\text { regarding supportive care in } \\
\text { childhood cancer }{ }^{19} \text {. }\end{array}$ & $\begin{array}{l}2017 \\
\text { Amesterdã } \\
\text { PUBMED }\end{array}$ & $\begin{array}{l}\text { Investigar quais cuidados de } \\
\text { suporte que os pacientes e } \\
\text { pais consideram mais } \\
\text { importantes. }\end{array}$ & $\begin{array}{l}\text { Estudo Qualitativo } \\
\mathrm{N}=29\end{array}$ & $\begin{array}{l}\text { Melhorar os cuidados de } \\
\text { suporte e o desenvolvimento de } \\
\text { diretrizes incorporando as } \\
\text { preferências dos pacientes e } \\
\text { dos seus familiares. }\end{array}$ \\
\hline $\begin{array}{l}\text { Coping with the diagnosis and } \\
\text { hospitalization of a child with } \\
\text { childhood cancer }{ }^{21}\end{array}$ & $\begin{array}{l}2015 \\
\text { Brasil } \\
\text { scopus }\end{array}$ & $\begin{array}{l}\text { Conhecer como os familiares } \\
\text { enfrentam a hospitalização } \\
\text { da criança com câncer. }\end{array}$ & $\begin{array}{l}\text { Estudo Qualitativo } \\
\mathrm{N}=10\end{array}$ & $\begin{array}{l}\text { Fornecer subsídios para o } \\
\text { cuidado de enfermagem a esse } \\
\text { público. }\end{array}$ \\
\hline $\begin{array}{l}\text { A experiência da família da } \\
\text { criança e/ou adolescente em } \\
\text { cuidados paliativos: flutuando } \\
\text { entre a esperança e a } \\
\text { desesperança em um mundo } \\
\text { transformado pelas perdas }{ }^{23} \text {. }\end{array}$ & $\begin{array}{l}2015 \\
\text { Brasil } \\
\text { SCIELO }\end{array}$ & $\begin{array}{l}\text { Compreender a experiência } \\
\text { da família da criança e/ou } \\
\text { adolescente em cuidados } \\
\text { paliativos. }\end{array}$ & $\begin{array}{l}\text { Estudo } \\
\text { Qualitativo, com } \\
\text { referencial teórico } \\
\text { Interacionismo } \\
\text { Simbólico } \\
\mathrm{N}=15\end{array}$ & $\begin{array}{l}\text { Contribuir para o } \\
\text { reconhecimento do sofrimento } \\
\text { da família, por parte dos } \\
\text { enfermeiros, na trajetória dos } \\
\text { cuidados paliativos, e no seu } \\
\text { enfrentamento para as } \\
\text { questões de luto e morte }\end{array}$ \\
\hline
\end{tabular}

FIGURA 2: caracterização conforme categorias encontradas. O impacto do cuidado paliativo da criança com câncer na dinâmica familiar Rio de Janeiro, RJ, Brasil, 2020.

Após a leitura dos resumos dos artigos a amostra final compreendeu 13 publicações sendo 7 (54\%) provenientes de periódicos internacionais e 5 (46\%) nacionais. Com a análise dos artigos foi possível identificar duas categorias que compreenderam: $\mathrm{O}$ impacto do cuidado paliativo da criança com câncer na dinâmica familiar e o cuidado da equipe multiprofissional, em especial dos enfermeiros, à família da criança com câncer frente aos cuidados paliativos. 


\begin{tabular}{|c|c|c|c|c|}
\hline Título & $\begin{array}{l}\text { Ano, Local, } \\
\text { Base }\end{array}$ & Objetivos & $\begin{array}{l}\text { Metodologia/ } \\
\text { participantes }\end{array}$ & Contribuições \\
\hline $\begin{array}{l}\text { The experience of providing } \\
\text { end of life care at a children's } \\
\text { hospice: a qualitative study }{ }^{20} \text {. }\end{array}$ & $\begin{array}{l}2017 \\
\text { Irlanda do } \\
\text { Norte } \\
\text { PUBMED }\end{array}$ & $\begin{array}{l}\text { Explorar as experiências do } \\
\text { pessoal da equipe de } \\
\text { cuidados que prestam } \\
\text { assistência ao final da vida. }\end{array}$ & $\begin{array}{l}\text { Estudo } \\
\text { Qualitativo } \\
\mathrm{N}=12\end{array}$ & $\begin{array}{l}\text { Melhorar a experiência do } \\
\text { cuidado da equipe com a } \\
\text { família em cuidados paliativos } \\
\text { de crianças dentro do cenário } \\
\text { agudo. }\end{array}$ \\
\hline $\begin{array}{l}\text { Cuidados paliativos em } \\
\text { oncologia pediátrica: } \\
\text { Percepções, saberes e práticas } \\
\text { na perspectiva da equipe } \\
\text { multiprofissional } 22 \text {. }\end{array}$ & $\begin{array}{l}2015 \\
\text { Brasil } \\
\text { LILACS }\end{array}$ & $\begin{array}{l}\text { Conhecer as percepções, } \\
\text { saberes e práticas da equipe } \\
\text { multiprofissional na atenção } \\
\text { às crianças em cuidados } \\
\text { paliativos. }\end{array}$ & $\begin{array}{l}\text { Estudo } \\
\text { Qualitativo } \\
\mathrm{N}=9\end{array}$ & $\begin{array}{l}\text { Enfatizar que no ensino e na } \\
\text { pesquisa o compartilhando de } \\
\text { experiências passa a incluir, } \\
\text { também, a equipe } \\
\text { multiprofissional. }\end{array}$ \\
\hline $\begin{array}{l}\text { A experiência da família da } \\
\text { criança e/ou adolescente em } \\
\text { cuidados paliativos: flutuando } \\
\text { entre a esperança e a } \\
\text { desesperança em um mundo } \\
\text { transformado pelas perdas }{ }^{23} \text {. }\end{array}$ & $\begin{array}{l}2015 \\
\text { Brasil } \\
\text { SCIELO }\end{array}$ & $\begin{array}{l}\text { Compreender a experiência } \\
\text { da família da criança e/ou } \\
\text { adolescente em cuidados } \\
\text { paliativos. }\end{array}$ & $\begin{array}{l}\text { Estudo } \\
\text { Qualitativo, com } \\
\text { referencial } \\
\text { teórico } \\
\text { Interacionismo } \\
\text { Simbólico } \\
\mathrm{N}=15\end{array}$ & $\begin{array}{l}\text { Reconhecer o sofrimento da } \\
\text { família, na trajetória dos } \\
\text { cuidados paliativos, e } \\
\text { posterirormente no seu } \\
\text { enfrentamento para as } \\
\text { questões de luto. }\end{array}$ \\
\hline $\begin{array}{l}\text { Criança com câncer em } \\
\text { processo de morrer e sua } \\
\text { família: Enfrentamento } \\
\text { da equipe de Enfermagem²4. }\end{array}$ & $\begin{array}{l}2015 \\
\text { Brasil } \\
\text { LILACS }\end{array}$ & $\begin{array}{l}\text { Descrever as } \\
\text { especificidades do cuidado } \\
\text { de enfermagem à criança } \\
\text { com câncer em processo de } \\
\text { morrer e sua família. }\end{array}$ & $\begin{array}{l}\text { Estudo } \\
\text { Qualitativo } \\
\mathrm{N}=6\end{array}$ & $\begin{array}{l}\text { Contribuir na capacitação da } \\
\text { equipe acerca dos cuidados } \\
\text { paliativos, o processo de } \\
\text { morrer, morte e luto. }\end{array}$ \\
\hline $\begin{array}{l}\text { Caring for pediatric patients' } \\
\text { families at the child's end of } \\
\operatorname{life}^{25} \text {. }\end{array}$ & $\begin{array}{l}2015 \\
\text { Estados } \\
\text { Unidos } \\
\text { CINAHAL }\end{array}$ & $\begin{array}{l}\text { Descrever um programa de } \\
\text { luto projetado para facilitar a } \\
\text { comunicação duradoura com } \\
\text { a família de luto. }\end{array}$ & $\begin{array}{l}\text { Estudo } \\
\text { Qualitativo } \\
\text { estudo de caso } \\
\mathrm{N}=1\end{array}$ & $\begin{array}{l}\text { Promover estratégias práticas } \\
\text { permitindo que os } \\
\text { enfermeiros se comuniquem } \\
\text { com a família da criança no } \\
\text { processo de morrer. }\end{array}$ \\
\hline $\begin{array}{l}\text { Palliative care in pediatric } \\
\text { patients with hematologic } \\
\text { Malignancies }^{26} \text {. }\end{array}$ & $\begin{array}{l}2015 \\
\text { Estados } \\
\text { Unidos } \\
\text { PUBMED }\end{array}$ & $\begin{array}{l}\text { Compreender as barreiras e } \\
\text { os benefícios da integração } \\
\text { de cuidados paliativos de } \\
\text { crianças com malignidades } \\
\text { hematológicas }\end{array}$ & $\begin{array}{l}\text { Estudo } \\
\text { Qualitativo } \\
\mathrm{N}=01\end{array}$ & $\begin{array}{l}\text { Reconhecer a necessidade de } \\
\text { atenção para a gestão de } \\
\text { sintomas, sofrimento espiritual } \\
\text { e emocional, e empatia com os } \\
\text { familiares. }\end{array}$ \\
\hline $\begin{array}{l}\text { Parents' experience with a } \\
\text { dying child with cancer in } \\
\text { palliative care }{ }^{27} \text {. }\end{array}$ & $\begin{array}{l}2015 \\
\text { Eslováquia } \\
\text { SCOPUS }\end{array}$ & $\begin{array}{l}\text { Esclarecer e analisar a } \\
\text { experiência de pais com falha } \\
\text { no tratamento e a morte de } \\
\text { seus filhos que sofrem de } \\
\text { câncer }\end{array}$ & $\begin{array}{l}\text { Estudo } \\
\text { Qualitativo } \\
\mathrm{N}=5\end{array}$ & $\begin{array}{l}\text { Ajudar enfermeiros e outros } \\
\text { profissionais de saúde a } \\
\text { entender melhor as questões da } \\
\text { perspectiva dos pais a cerca da } \\
\text { falha do tratamento }\end{array}$ \\
\hline
\end{tabular}

FIGURA 3: Caracterização conforme categorias encontradas. O impacto do cuidado paliativo da criança com câncer na dinâmica familiar Rio de Janeiro, RJ, Brasil, 2020.

\section{DISCUSSÃO}

\section{O impacto do cuidado paliativo da criança com câncer na dinâmica familiar}

Nos artigos ${ }^{14-21}$ foi evidenciado que as famílias passam por momentos conflituosos diante do diagnóstico de câncer no seu filho, bem como a evolução para os cuidados paliativos exclusivos. Elas tentam se adaptar à nova condição de saúde-doença do seu filho em cuidados paliativos que pode ser representado, sobretudo, pelo sentimento de esperança, desespero, culpa, negação, revolta e medo da morte. Essas informações foram extraídas dos artigos supracitados e serão descritas no decorrer dessa discussão.

A experiência começa com a família tendo a vida estilhaçada, caracterizada pela notícia de que a criança e/ou adolescente foi diagnosticada com câncer e evoluiu para os cuidados paliativos. Essa modalidade de tratamento expõe a família à uma nova realidade: o tratamento medicamentoso existente não é suficiente para impedir ou controlar o avanço da doença ${ }^{20}$.

Nesse sentido, o impacto na família está relacionado com longos períodos de hospitalização, reinternações frequentes, terapêutica agressiva, dificuldade de separação da família, limitações na compreensão do diagnóstico, angústia, dor e sofrimento. As dificuldades para desempenhar esse papel são situações de enfrentamento vivenciado pelos familiares nesse processo de adaptação diante da doença e de possível luto diante da morte da criança ${ }^{27}$. 
Ao viver a experiência de ter um filho em cuidados paliativos, apesar do sentimento de perda estar presente em todo o percurso da doença, só tende a ser reconhecidos de fato após a morte, quando se perde a relação única, que é percebida como vital por quem a vivencia. Manejar esses sentimentos, antecipadamente, é difícil e assustador para os pais com filhos em fase final de vida ${ }^{28}$.

Nesse contexto, reconhecer os cuidados paliativos é uma tarefa árdua para a família, uma vez que a coloca diante das incertezas e apreensão em relação ao futuro e a possibilidade da morte ${ }^{20}$.

\section{O cuidado da equipe multiprofissional, em especial dos enfermeiros, à família da criança com câncer frente aos} cuidados paliativos

Os estudos ${ }^{22-26}$ demonstram como a equipe multiprofissional, principalmente, os enfermeiros se agregam com o mesmo objetivo de fornecer uma assistência digna e humanizada para a criança com câncer em cuidados paliativos, priorizando a família como parte integral dessa modalidade de tratamento.

Diante da complexidade da assistência em oncologia pediátrica, destaca-se a importância de uma equipe de saúde multiprofissional. A enfermagem, como integrante dessa equipe, está presente nas diferentes etapas de cuidado, desde a prevenção, o diagnóstico, os tratamentos prolongados, até o cuidado paliativo ${ }^{29}$. Este cuidado é potencializado ante o diagnóstico de câncer sem possibilidades terapêuticas. O enfermeiro se torna referência para o apoio à criança e ao adolescente, bem como à sua família para enfrentar a fase terminal e, consequentemente, a morte ${ }^{30}$.

Sendo assim, a abordagem da equipe interdisciplinar no câncer pediátrico representa para o cuidado à base da maior parte da oncologia moderna e do progresso futuro. É uma área de oncologia que tem visto resultados notáveis, com avanços terapêuticos que têm aumento das taxas de sobrevida para alguns tipos de câncer, mas também apresenta desafios devastadores em que alguns cânceres pediátricos permanecem incuráveis ${ }^{17}$.

Conhecer as concepções relacionadas à implementação dos cuidados paliativos, assim como o processo de finitude, possibilitaria aos enfermeiros uma melhor compreensão dos seus valores e crenças diante desse processo, sentindo-se preparados no momento em que atuassem lidando com pacientes e familiares que estariam nessa situação ${ }^{31}$.

Dessa forma, o profissional que trabalha em oncologia pediátrica depara-se inicialmente com uma importante mudança de foco na atenção. Ele que é preparado para lidar com o conceito de saúde, passa a trabalhar com o conceito de doença em progressão. A almejada cura começa a dar lugar à busca da qualidade de vida ${ }^{5,32}$.

Assim, a mesma equipe que sofre as ambivalências de sua práxis profissional, também consegue compreender a importância de sua atuação nesses momentos de sofrimento e sente-se gratificada em poder garantir à criança uma vida digna até o momento de sua morte ${ }^{28}$.

O modelo de assistência geralmente adotado nas unidades de terapia intensiva, pautado no intervencionismo e no curativismo, dificulta o cuidado em todas as suas dimensões, ou seja, atendendo às diversas necessidades do paciente, independente da fase de vida em que ele se encontra. Na perspectiva da terminalidade, a utilização dos mais sofisticados recursos para a manutenção da vida não será suficiente a esse paciente ${ }^{8,33}$.

Nesse contexto, entram em prática os cuidados paliativos, os quais, embora ainda pouco utilizados em pacientes terminais de UTIs, são considerados alvos de estudos, especialmente os critérios para a sua aplicação. Com isso, tornase importante a aceitação da morte, interpretando-a como um evento natural, sem descuidar do respeito à autonomia e não maleficência do paciente $e^{9,34}$.

A equipe de enfermagem, como parte indispensável da equipe multidisciplinar na oncologia pediátrica, é mentora de várias funções no contexto do seu dia a dia, estando presente desde o início, com a descoberta do diagnóstico, bem como participando dos transtornos da criança e da família, tornando-as capazes de conhecer e ter uma ampla visão de todas as suas necessidades 35,36 .

\section{Limitações do estudo}

Como limitação do estudo acredita-se que o recorte temporal empregado na estratégia de busca das publicações, pode ter contribuído para a exclusão de pesquisas sobre a temática publicadas antes ou depois deste período. Além disso, mesmo utilizando diferentes bases de dados para encontrar artigos que abordassem os cuidados paliativos exclusivos na unidade de terapia intensiva, não foi possível identificá-los. Nesse sentindo demonstra-se que existe uma lacuna do conhecimento acerca desse tema.

\section{CONCLUSÃO}

De acordo com as categorias encontradas foi possível identificar que o tema cuidados paliativos vem ganhando notoriedade na prática de ensino e saúde, em âmbito nacional e internacional. Nesse contexto todos os envolvidos no processo de cuidar, família e equipe multiprofissional, buscam, de alguma forma, minimizar o sofrimento e a angústia 
advindos da doença em estágio avançado. Nesse âmbito, a assistência de enfermagem está direcionada em promover qualidade de vida para crianças em cuidados paliativos no tempo de vida que restam, bem como para as famílias.

Dessa forma, os cuidados de suporte preenchem uma importância cada vez maior no planejamento do desenvolvimento de diretrizes com incorporação de tomada de decisão compartilhada, por parte da família e da equipe multiprofissional no que tange a criança em cuidados paliativos.

Sendo assim, em situações de crise são esperadas mudanças nos papéis tanto dos familiares que são desafiados a aceitar e enfrentar que seu filho está em cuidados paliativos e iminência de morte, como, também, dos enfermeiros que reconhecem que o tratamento da criança deve ser abrangente, individualizada e humanizada pautadas na comunicação honesta.

Destaca-se, ainda, a escassez de produções do conhecimento acerca dos cuidados paliativos à criança com câncer em unidades de terapia intensiva, pontuando a necessidade de novos estudos sobre tema tão relevante para os enfermeiros que atuam na assistência direta com esse público.

\section{REFERÊNCIAS}

1. Instituto Nacional de Câncer José Alencar Gomes da Silva. Câncer infanto-juvenil. Rio de Janeiro: Ministério da Saúde [Internet]. 2019 [cited 2020 Mar 01]. Available from: https://www.inca.gov.br/tipos-de-cancer/cancer-infantojuvenil.

2. Instituto Nacional do Câncer José Alencar Gomes da Silva. Estimativas/2018: Incidência de Câncer no Brasil. Rio de Janeiro: Ministério da Saúde [Internet]. 2019. [cited 2020 Mar 01]. Available from: http://www1.inca.gov.br/rbc/n_64/v01/pdf/15resenha-estimativa-2018-incidencia-de-cancer-no-brasil.pdf.

3. Fermo VC, Lourençatto GN, Medeiros TS, Anders JC, Souza AlJ. Early diagnosis of child cancer: the journey taken by families. Esc Anna Nery [Internet]. 2014 [cited 2020 Jan 01]; 18(1): 54-9. Available from: https://www.scielo.br/pdf/ean/v18n1/14148145-ean-18-01-0054.pdf.

4. World Health Organization-WHO. Palliative Care Geneva: WHO; [Internet]. 2017 [cited 2020 Mar 07]. Available from: http://www.who.int/mediacentre/factsheets/fs402/en/.

5. França JRFS, Costa SFG, Andrade CG, Costa ICP, Souza ATO, Souto MC. Nurses' experience in caring for a terminally ill child: study in the light of the humanistic nursing theory. Cienc Cuid Saúde [Internet]. 2014 [cited 2020 Jan 10]; 13(3):425-32. DOI: https://doi.org/10.4025/cienccuidsaude.v13i3.17139.

6. Garcia-Schinzari NR, Sposito AMP, Pfeifer LI. Palliative care in hospitalized children and adolescents with cancer: the role of occupational therapy. Rev brasileira cancerol [Internet]. 2013 [cited 2020 Jan 10]; 59(2):239-47. Available from: https://rbc.inca.gov.br/site/arquivos/n_59/v02/pdf/11b-cuidados-paliativos-junto-a-criancas-e-adolescentes-hospitalizadoscom-cancer-o-papel-da-terapia-ocupacional.pdf.

7. Brito MA, Soares EO, Rocha SS, Figueiredo MLF. Palliative care in pediatrics: a reflective study. Rev enferm UFPE [Internet]. 2015 [cited 2020 Jan 22]; 9(3):7155-60. Available from: https://periodicos.ufpe.br/revistas/revistaenfermagem/article/view/10446/11259.

8. Almeida LF. Terminalidade humana na UTI: reflexões sobre a formação profissional e ética diante da finitude. Rev enferm HUPE [Internet]. 2013 [cited 2020 Jan 22]; 12(3):147-53. Available from: https://www.epublicacoes.uerj.br/index.php/revistahupe/article/view/7542.

9. Hagstrom S. Family stress in pediatric critical care. J Pediatr Nurs [Internet]. 2017 [cited 2020 Dec 10]; 32:32-40. DOI: https://doi.org/10.1016/j.pedn.2016.10.007.

10. Soares CB, Hoga LAK, Peduzzi M, Sangaleti C, Yonekura T, Silva DRAD. Integrative review: concepts and methods used in nursing. Rev Esc Enferm USP [Internet]. 2014 [cited 2020 Jan 22]; 48(2):335-45. Available from: http://www.scielo.br/pdf/reeusp/v48n2/pt_0080-6234-reeusp-48-02-335.pdf.

11. Botelho LLR, Cunha CCA, Macedo M. The integrative review method in organizational studies. Gestao Soc [Internet]. 2011[cited 2020 Feb 05]; 5(11):121-36 Available from: http://www.gestaoesociedade.org/gestaoesociedade/article/view/1220.

12. The Joanna Briggs Institute (JBI). Joanna Briggs Institute Reviewers' Manual: 2014 edition. Adelaiade: JBI [Internet]. 2014 [cited 2020 Feb 05]; Available from: https://nursing.Isuhsc.edu/JBI/docs/ReviewersManuals/Economic.pdf.

13. Galvão TF, Pansani, TSA, Harrad D. Principais itens para relatar revisões sistemáticas e meta-análises: a recomendação PRISMA. Epidemiol Serv Saude [Internet]. 2015 [cited 2020 Feb 05]; 24(2):335-42. Available from: http://www.scielo.br/pdf/ress/v24n2/2237-9622-ress-24-02-00335.pdf.

14. Assis LA, Batista PSS, Alves DR, et al. Mothers' experience of children with cancer under palliative care. Rev Fun Care Online [Internet]. 2020 [cited 2020 Mar 05]; 12:1348-54. DOI: http://dx.doi.org/10.9789/2175-5361.rpcfo.v12.9450.

15. Hendricks-Ferguson, Verna L. PhD, RN, CHPPN, FPCN, FAAN; Haase, Joan E. PhD, RN, FAAN. Parent perspectives of receiving early information about palliative and end-of-life care options from their child's pediatric providers. Cancer Nursing [Internet]. 2019 [cited 2020 Mar 05]; 42(4):22-30. Doi: https://doi.org/10.1097/NCC.0000000000000589.

16. Kaye EC, DeMarsh S, Gushue CA, Jerkins J, Sykes A, Lu Z, Snaman JM, et al. Predictors of location of death for children with cancer enrolled on a palliative care service. The Oncologist [Internet]. 2018 [cited 2020 Feb 05]; 23:1525 -32. Available from: https://www.ncbi.nlm.nih.gov/pubmed/29728467. 
17. Costa MADJ, Agra G, Santos NCCB, Oliveira CDB, Freire MEM, Costa MML. Experiences of the mothers of children with cancer in palliative care. Rev enferm UFPE on line [Internet]. 2018 [cited 2020 Feb 05]; 12(5):1355-64. Available from: https://periodicos.ufpe.br/revistas/revistaenfermagem/article/view/235877.

18. Tenniglo LJA, Loeffen EAH, Kremer LCM, Font-Gonzalez A, Mulder RL, Postma A, et al. Patients' and parents' views regarding supportive care in childhood cancer. Supportive Care in Cancer [Internet]. 2017 [cited 2020 Feb 05]; 25(10):3151-60. Available from: https://link.springer.com/article/10.1007/s00520-017-3723-7\#citeas.

19. Noia TC, Sant'Ana RSE, Santos ADS, Oliveira SC, Bastos Veras SMC, Lopes-Júnior LC. Coping with the diagnosis and hospitalization of a child with childhood cancer. Invest Educ Enferm [Internet]. 2015 [cited 2020 Feb 12]; 33(3):465-72. Available from: http://www.scielo.org.co/scielo.php?script=sci_arttext\&pid=S012053072015000300010\&lng=en\&nrm=iso\&tlng=pt.

20. Misko MD, Santos MR, Ichikawa CRF, Lima RAG, Bousso RS. A experiência da família da criança e/ou adolescente em cuidados paliativos: flutuando entre a esperança e a desesperança em um mundo transformado pelas perdas. Rev. latinoam. Enfermagem [Internet]. 2015 [cited 2020 Feb 12]; 23(3): 560-7. Available from: http://www.scielo.br/pdf/rlae/v23n3/pt_01041169-rlae-23-03-00560.pdf.

21. Gurková E, Andraščíková I, Cap J. Parents' experience with a dying child with cancer in palliative care. Cent Eur J Nurs Midw [Internet]. 2015 [cited 2020 Mar 07]; 6(1):201-8. Available from: http://periodika.osu.cz/cejnm/dok/2015-01/4-gurkovaandrascikova-cap.pdf.

22. McConnell T, Porter S. The experience of providing end of life care at a children's hospice: a qualitative study. BMC Palliat Care [Internet]. 2017 [cited 2020 Feb 05]; 16. Available from: https://www.ncbi.nlm.nih.gov/pmc/articles/PMC5307784/.

23. Silva AF, Issi HB, Motta MGC, Botene DZA. Palliative care in paediatric oncology: perceptions, expertise and practices from the perspective of the multidisciplinary team. Rev Gaúcha Enferm [Internet]. 2015 [cited 2020 Feb 12]; 36(2):56-62. Available from: http://www.scielo.br/pdf/rgenf/v36n2/pt_1983-1447-rgenf-36-02-00056.pdf.

24. Carmo SA; Oliveira ICS. Children with cancer in the process of dying and their families: confrontation of nursing team. Rev Bras de Cancerol [Internet]. 2015 [cited 2020 Feb 12]; 61(2):131-8. Available from: http://www1.inca.gov.br/rbc/n_61/v02/pdf/07artigo-crianca-com-cancer-em-processo-de-morrer-e-sua-familia-enfrentamento-da-equipe-de-enfermagem.pdf.

25. Mullen JE, Reynolds MR, Larson JS. Caring for pediatric patients' families at the child's end of life. Critical Care Nurse [Internet]. 2015 [cited 2020 Feb 12]; 35(6):46-56. Available from: http://ccn.aacnjournals.org/content/35/6/46.full.

26. Humphrey L, Kang TI. Palliative care in pediatric patients with hematologic Malignancies. Hematology [Internet]. 2015 [cited 2020 Feb 12]; 1:490-95. Available from: http://asheducationbook.hematologylibrary.org/content/2015/1/490.

27. Kreicbergs UC, Lannen $P$, Onelov E, Wolfe J. Parental grief after losing a child to cancer: impact of professional and social support on long-term outcomes. J Clin Oncol [ Internet]. 2007 [cited 2020 Dec 10]; 25(22):3307-12. DOI: https://doi.org/10.1200/JCO.2006.10.0743.

28. Bousso, Regina Szylit. Um tempo para chorar: a família dando sentido à morte prematura do filho [livre docência]. São Paulo: Universidade de São Paulo, Escola de Enfermagem [Tese de Doutorado], 2006 [cited 2020 Dec 10]. DOI: https://doi.org/10.11606/T.7.2006.tde-27092006-104500.

29. Mutti CF, Padoin SMM, Paula CC. Spaciality of being-nursing-professional in the world of caring to children who has cancer. Esc. Anna Nery [Internet]. 2012; [cited 2020 Dec 10]; 16(3):493-9. Available from: https://www.scielo.br/scielo.php?pid=S1414$81452012000300010 \&$ script $=$ sci_abstract\&tlng=pt.

30. Avanci BS, Carolindo FM, Góes FGB, Netto NPC. Palliative care to the onchologic child in the situation of live / die: the optics of the care in nursing. Esc. Anna Nery [Internet]. 2009 [cited 2020 Dec 10]; 13(4):708-16. Available from: https://www.scielo.br/scielo.php?pid=S1414-81452009000400004\&script=sci_abstract\&tlng=pt.

31. Santos MCL, Pagliuca LMF, Fernandes AFC. Palliative care to the cancer patient: reflections according to Paterson and Zderad's view. Rev. latinoam. Enferm. [Internet], 2007 [cited 2020 Mar 01]; 15(2). Available from: http://www.scielo.br/pdf/rlae/v15n2/pt_v15n2a24.pdf.

32. Smith MA, Altekruse SF, Adamson PC, Reaman GH, Seibel NL. Declining childhood and adolescent cancer mortality. Cancer [Internet]. 2014 [cited 2020 Mar 01]; 120:2497-506. Available from: https://www.ncbi.nlm.nih.gov/pubmed/24853691.

33. 33 Vieira A.P.M.S, Castro D.L, Coutinho MS. Assistência de enfermagem na oncologia pediátrica. Rev. Eletrôn. Atualiza Saúde [Internet]. 2016 [cited 2020 Dec 10]; 3(3):67-75. Available from: http://atualizarevista.com.br/wpcontent/uploads/2016/01/Assist\%C3\%AAncia-de-enfermagem-na-oncologia-pedi\%C3\%A1trica-v-3-n-3.pdf.

34. Silveira NR, et al. Cuidado paliativo e enfermeiros de terapia intensiva: sentimentos que ficam. Rev Bras Enferm [Internet]. 2016 [cited 2020 Dec 10]; (6):1074-81. Available from: https://www.scielo.br/pdf/reben/v69n6/0034-7167-reben-69-06-1074.pdf.

35. Germano KS, Meneguin S. Meanings attributed to palliative care by Nursing undergraduates. Acta paul enferm [Internet]. 2013 [cited 2020 Dec 10]; 26(6):522-8. Available from: http://www.scielo.br/scielo.php?pid=S010321002013000600003\&script=sci_arttext.

36. Das K, Khanna T, Arora A, Agrawal N. Parents' acceptance and regret about end of life care for children who died due to malignancy. Supportive Care in Cancer [Internet]. 2020 [cited 2020 Mar 05]; 28:303-8. Available from: https://doi.org/10.1007/s00520-019-04806-0. 